\title{
A new optical technique to monitor joint motion using position sensitive detector
}

\author{
Gu-In Junga, ${ }^{\mathrm{a}, 1}$, Byung Kyu Park ${ }^{\mathrm{b}, 1}$, Ji-Sun Kim ${ }^{\mathrm{a}}$, Tae-Hee Lee ${ }^{\mathrm{a}}$, Ju-Hyeon Choi ${ }^{\mathrm{a}}$, \\ Han-Byeol Oh ${ }^{\mathrm{a}}$, A-Hee Kimª ${ }^{\mathrm{a}}$, Bong-Jun Goh ${ }^{\mathrm{a}}$, Ji-Won Kim ${ }^{\mathrm{a}}$, Ki Sung Lee ${ }^{\mathrm{c}}$ and \\ Jae-Hoon Jun ${ }^{\text {a,* }}$ \\ ${ }^{a}$ Department of Biomedical Engineering, BK21+ Research Institute of Biomedical Engineering, \\ College of Biomedical and Health Science, Konkuk University, Chungju, Korea \\ ${ }^{\mathrm{b}}$ Korea University College of Medicine, Seoul, Korea \\ ${ }^{c}$ Yon-Sei Family Clinic, Kimpo, Korea
}

\begin{abstract}
.
BACKGROUND: Evaluation of joint movements is essential to choose an appropriate rehabilitation protocol for a patient. OBJECTIVE: The aim of this study is to suggest an alternative optical technique for measurement of joint angle which is convenient, inexpensive, and can be operated in real time.

METHODS: According to the principle of the triangulation method, position sensitive detector (PSD) converts reflected light signals into distance-related voltages. Various parameters were investigated to increase detection range and resolution of joint angle measurements.

RESULTS: The accuracy of the suggested optical sensor was verified by comparing with a commercial goniometer and 3D motion capture system.

CONCLUSIONS: This method can be applied to monitor recovery progress for the patient in rehabilitation and sport science.
\end{abstract}

Keywords: Human joint angle, optical goniometer, position sensitive detector, rehabilitation, range of motion

\section{Introduction}

Recently, population ageing, safety accidents, and excessive exercises have increased the number of patients with functional disorders [1]. For these reasons, rehabilitation is concerned with the development and restoration of human movement and functionalities after injury.

In general, measured functional parameters are muscular function, endurance, movement patterns, and range of motion (ROM) of the joint in the rehabilitation field [2]. Variations in the motion of joint have historically enabled physicians to establish a diagnosis and then monitor the pathological state. Furthermore, ROM measurements have been used as a basic marker for normal or disability [3].

The goniometry is one of the human joint angle measurement tools that evaluate a patient's ROM [4]. Physiotherapists measure the initial state of the patient and monitor joint angle changes prior to and after

\footnotetext{
${ }^{1}$ These authors contributed equally to this work.

${ }^{*}$ Corresponding author: Jae-Hoon Jun, Department of Biomedical Engineering, College of Biomedical and Health Science, Konkuk University, 268 Chungwondaero, Chungju, Chungbuk 380-701, Korea. Tel.: +82 43840 3799; E-mail: jjun81@ kku.ac.kr.
} 


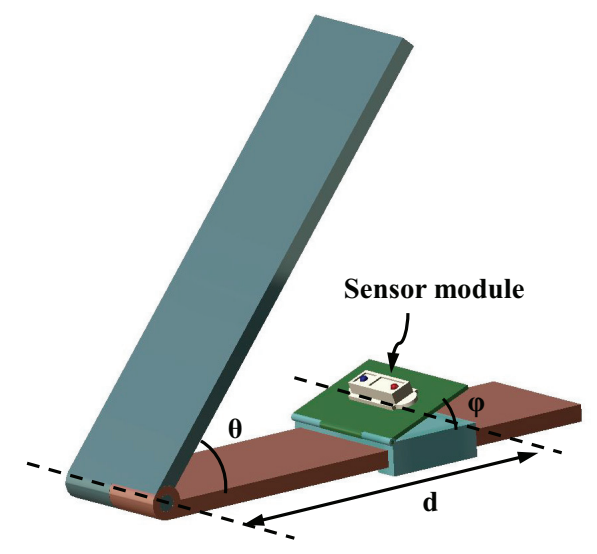

Fig. 1. Joint model and the experimental setup used in this study.

the treatment. Therefore, an accurate measurement of the joint movement is an important matter in the rehabilitation process.

Conventional electrogoniometer has a drawback of insufficient accuracy due to the abrasion of internal resistance caused by repeated uses [5]6]. The camera-based optical motion analysis systems offer the most precise evaluation of human motion, but the equipment is very expensive and can only be used in a specialized motion laboratory with specialists for data analysis [7 [8]. An optical method, based on the intensity variation of the optical signal through the fiber when subjected to macrobending, has been reported for joint angle measurement. However, it was unstable and inaccurate due to undesirable bends and fluctuations in the optical power and hysteresis. Also, this technique was not durable for repeated uses [9].

In this paper, a new optical sensor was developed for monitoring the movement of human joint with position sensitive detector. Various sensor variables were investigated to find the best combination of parameters for wide detection range and resolution. The appropriate mathematical model was obtained with detected output signals to measure joint angle changes in real time and to display on laptop monitor. The accuracy of the suggested sensor was evaluated by comparing with commercial goniometer and 3D motion capture system.

\section{Materials and methods}

Figure 1 shows the suggested joint angle sensor using an optical method. The sensor module (GP2Y0A41SK0F, sharp, Japan) is a distance measuring sensor unit, composed of an integrated combination of position sensitive detector (PSD), infrared emitting diode (IRED), and signal processing unit. The constant voltage circuit is installed for stable signal output, and visible light is filtered out to prevent external light noise. The detecting distance range is 40 to $300 \mathrm{~mm}$. It provides an analog output voltage that is proportional to the distance variation. Typical average current consumption is $12 \mathrm{~mA}$.

PSD is a photo-diode detector to measure the position of the light spot based on the triangulation principle. The light illuminated on the detector is converted into an electrical current that is divided between the contacts in proportion to the resistance of the active layer from the position of the incident light spot to the contacts. By the relative magnitude of the electric current from each contact point, the position can be determined [10]. This detector provides high resolution and a rapid response which is particularly suitable for applications where tiny displacements are required to be measured in real time. 


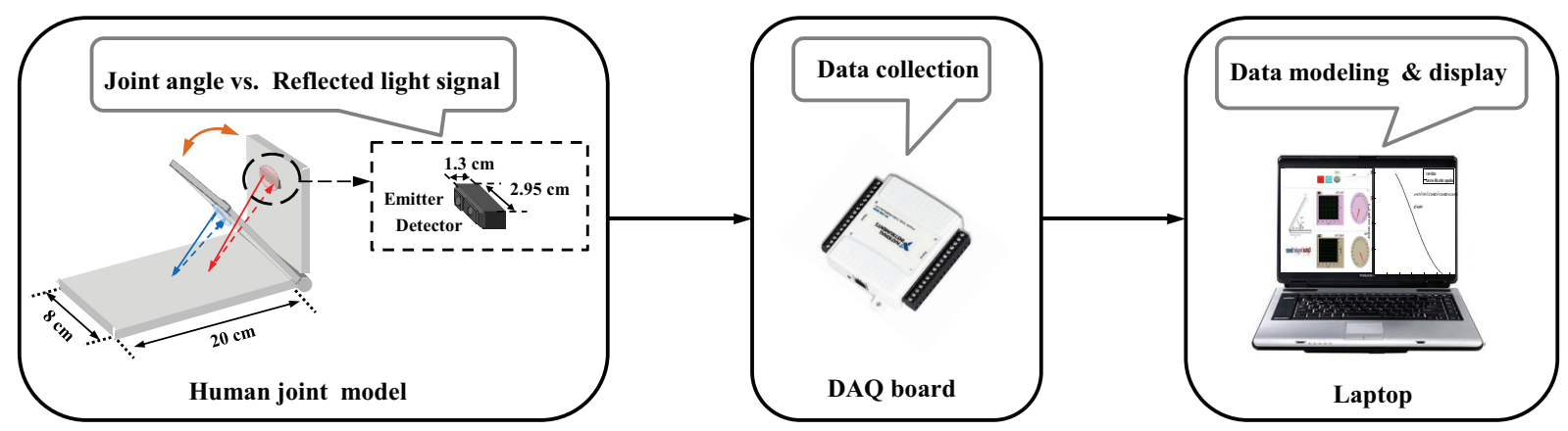

Fig. 2. The schematic drawing of the experimental procedure.

As the joint angle $(\theta)$ is changed, the distance between the sensor module and the reflector is changed which results in the changes of output light signals. Thus, the angular displacement can be measured by detecting voltage outputs from reflected light signals. With the developed joint angle sensor, various distances between the sensor module and the center of the joint model $(d=6 \mathrm{~cm} \sim 9 \mathrm{~cm})$, attached sensor's angles $\left(\varphi=70^{\circ} \sim 90^{\circ}\right)$, and joint angles $\left(\theta=20^{\circ} \sim 180^{\circ}\right)$ were applied to find the optimal combination of these variables for a wide detection range with the minimum error.

As shown in Fig. 2, output signals from the sensor were collected using DAQ board (NI USB-6008, National Instrument, USA) in digital form, and saved in a computer with LabVIEW 8.0 (National Instruments, USA). Modeling of the experimental data was done using Matlab program (R2008a, Mathworks, USA). Inverse models with polynomial equations were tested to measure the joint angle in real time. First, the relationship between PSD output and joint angle was mathematically modeled. Next, the inverse relationship was used to obtain the angle directly. The inverse model is possible when the functions show monotonic pattern such as the increase or decrease [5]. Using the obtained model, the joint angle change can be calculated and displayed with a laptop in real time.

\section{Results and discussion}

Figure 3 shows the change of the light signal by the joint angle changes $\left(\theta=20^{\circ} \sim 180^{\circ}\right)$ under different distances between the sensor module and the center of the joint model $(d=6 \mathrm{~cm} \sim 9 \mathrm{~cm})$. Similar experiments had been repeated at different attached sensor's angles $\left(\varphi=70^{\circ} \sim 90^{\circ}\right)$. With the exception of $\varphi=90^{\circ}$, the inflection points were observed for other cases $\left(\varphi=70^{\circ}, 75^{\circ}, 80^{\circ}, 85^{\circ}\right)$. The detected light signals have different peak patterns and beam profiles due to the different $d$ and $\varphi$. Thus, these two variables must be considered for developing an efficient sensor for joint motions.

Figure 4 shows the beam pattern changes by the joint angle with different distances between the sensor module and the center of the joint model $(d)$ and attached sensor's angles $(\varphi)$. These results describe the effect of distance between the sensor module and the center of the joint model $(d=7.5 \mathrm{~cm} \sim 9 \mathrm{~cm})$ on detected light signal with the attached sensor's angles, $\varphi=70^{\circ} \sim 90^{\circ}$. Figures 4 (a) and (b) show results in the range of $\varphi=70^{\circ} \sim 75^{\circ}$. The increase and decrease regions in all distances between the sensor module and the center of the joint model are observed. Figure 4(c) shows the result at $\varphi=80^{\circ}$. The inflection points are observed in the range of $d=7.5 \mathrm{~cm} \sim 8.5 \mathrm{~cm}$, but the light signal is decreased monotonically at $d=9 \mathrm{~cm}$. In Fig. 4(d), the inflection points are observed in the range of $d=7.5 \mathrm{~cm}$ $\sim 8 \mathrm{~cm}$, but the output signals decrease monotonically in the range of $d=8.5 \mathrm{~cm} \sim 9 \mathrm{~cm}$. As shown in 


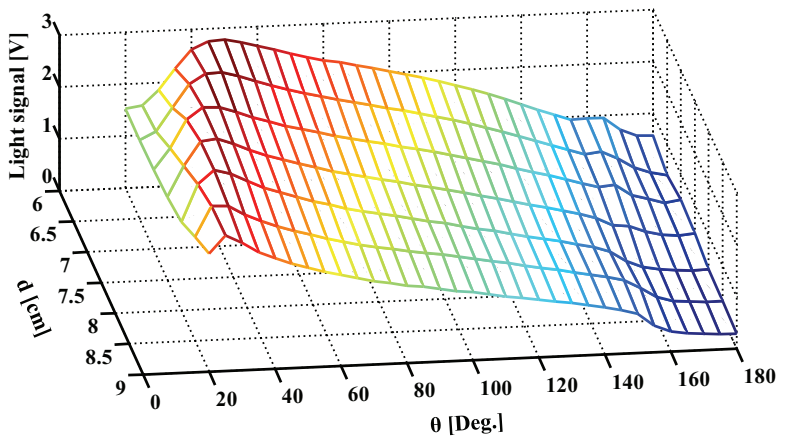

(a) $\varphi=70^{\circ}$

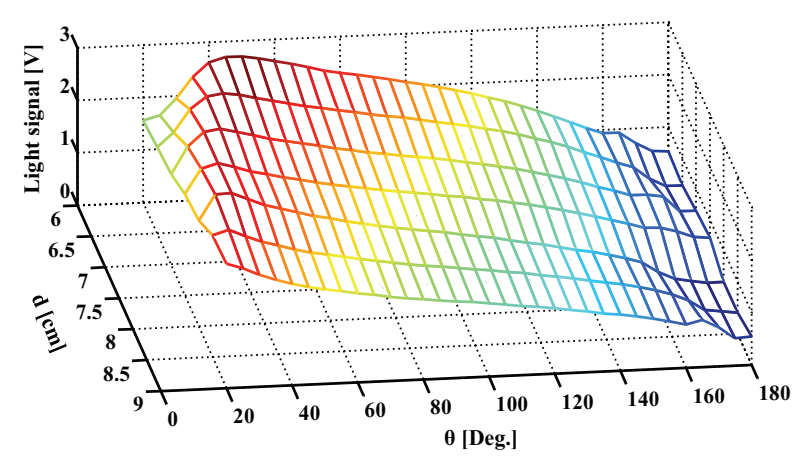

(c) $\varphi=80^{\circ}$

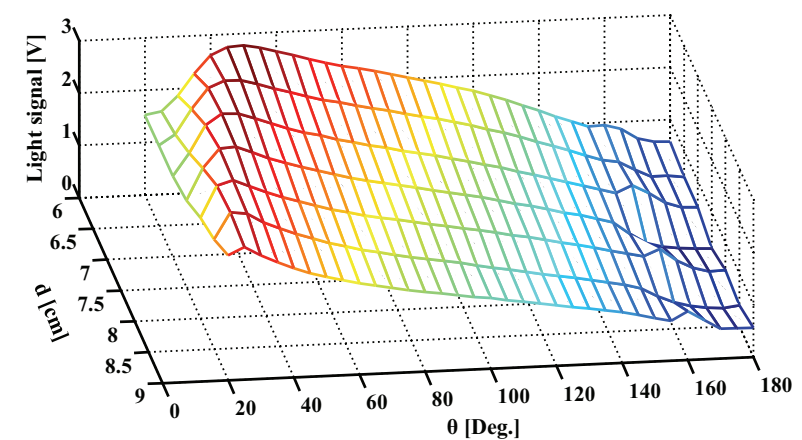

(b) $\varphi=75^{\circ}$

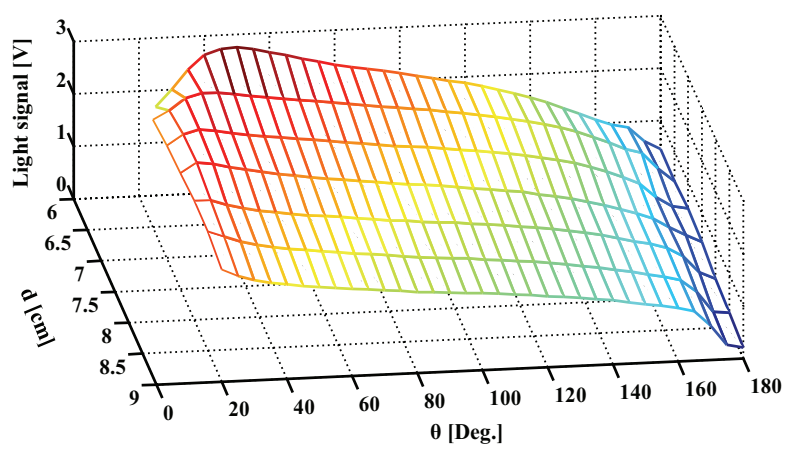

(d) $\varphi=85^{\circ}$

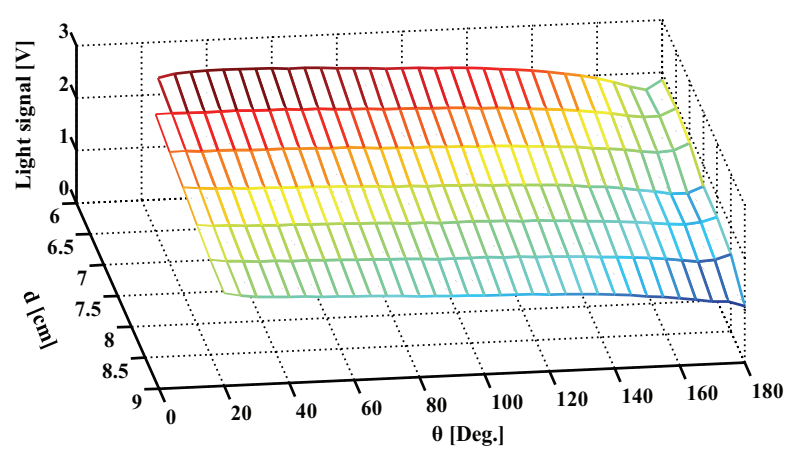

(e) $\varphi=90^{\circ}$

Fig. 3. The change of light signal by joint angle $(\theta)$.

Fig. 4(e), the output signals decrease monotonically in all cases. However, the resolution of the output signals is not sufficient for joint angle measurement.

The overall results are summarized as follows. As $d$ and $\varphi$ are increased, the value of the peak point is decreased and the inflection points shift the left side in Fig. 4. These results indicate that the measuring range, which can be described as the distance between inflection point (or peak value in light signals) and dashed line in Fig. 4, is increased. However, in an extreme case $\left(\varphi=90^{\circ}\right)$, the resolution is not good enough to measure joint angles.

Figure 5 shows the change of the measuring range by the sensor's attached angle and attached distance. In the case of $\varphi=90^{\circ}$, the resolution is not sufficient for determining the joint angle, so this case is 


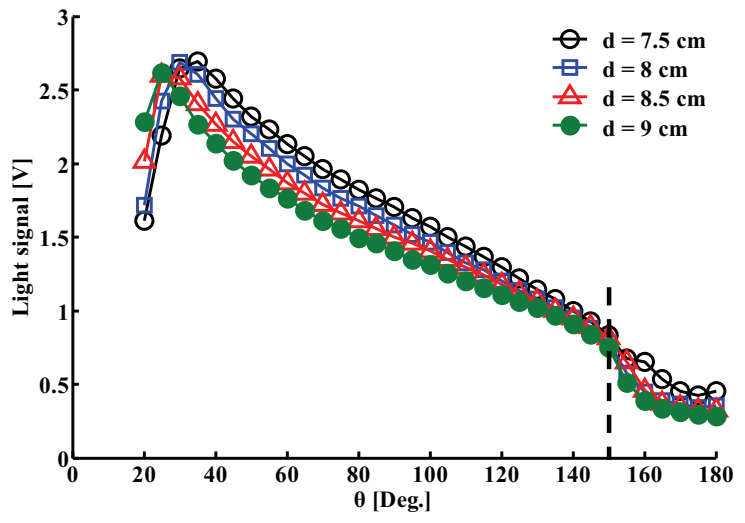

(a) $\varphi=70^{\circ}$

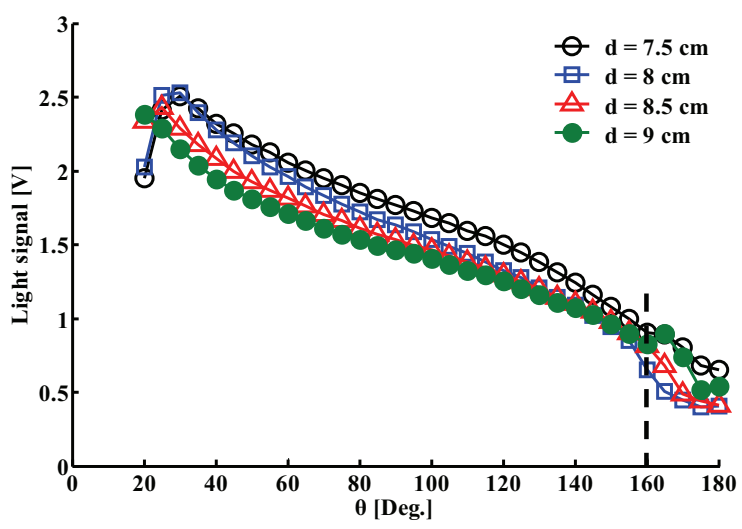

(c) $\varphi=80^{\circ}$

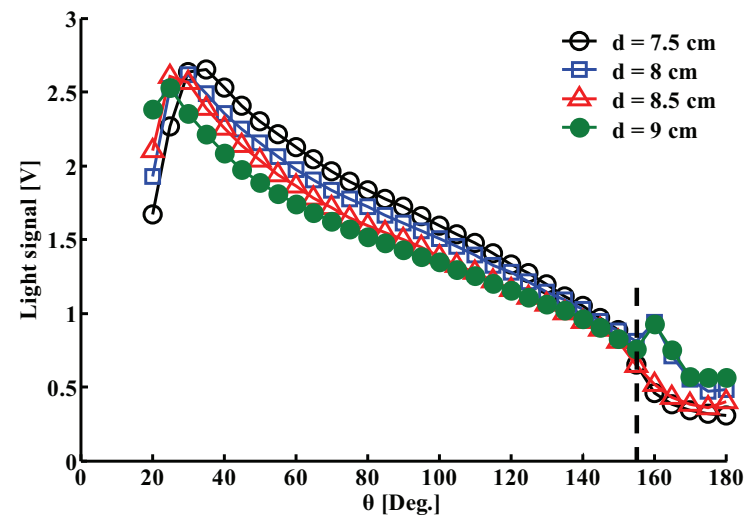

(b) $\varphi=75^{\circ}$

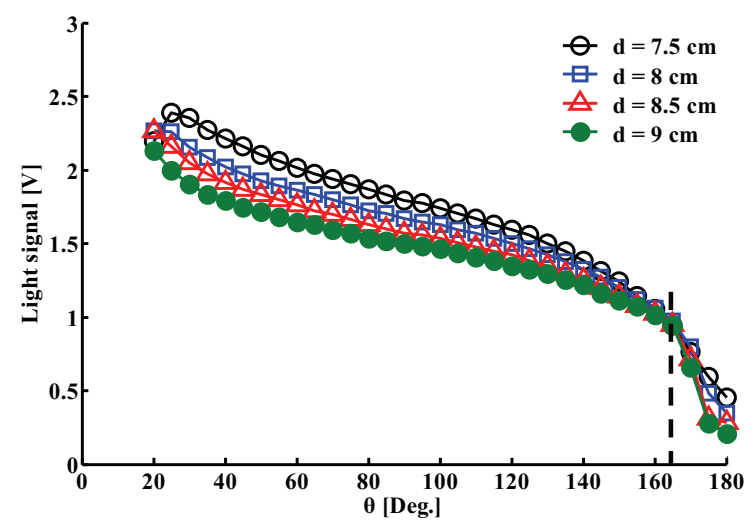

(d) $\varphi=85^{\circ}$

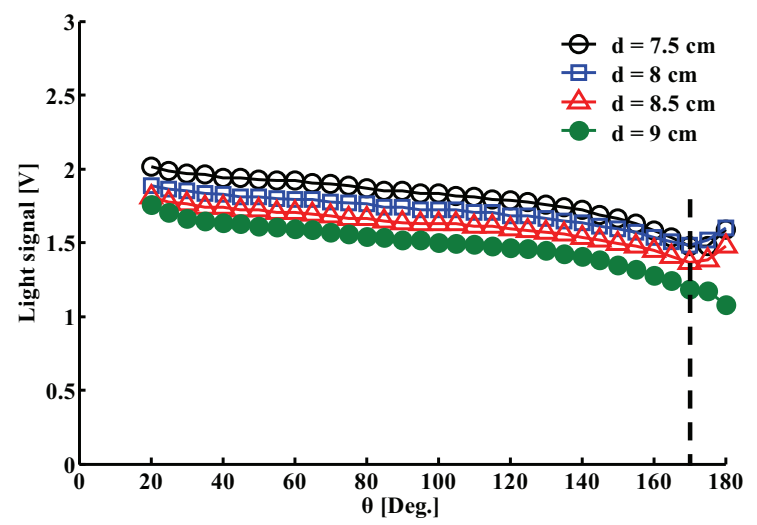

(e) $\varphi=90^{\circ}$

Fig. 4. The beam pattern changes by the sensor's attached distance $(d)$ and attached angle $(\varphi)$.

excluded. As $d$ and $\varphi$ are increased, the measuring range is increased. For $d=8.5 \mathrm{~cm}$ and $d=9 \mathrm{~cm}$ at $\varphi=85^{\circ}$, the similar measuring ranges are observed. However, the resolution is low for $d=9 \mathrm{~cm}$ as shown in Fig. 4(d). The best combination is found at $\varphi=85^{\circ}$ and $d=8.5 \mathrm{~cm}$; thus this combination is applied to mathematical modeling for joint angle measurements. 


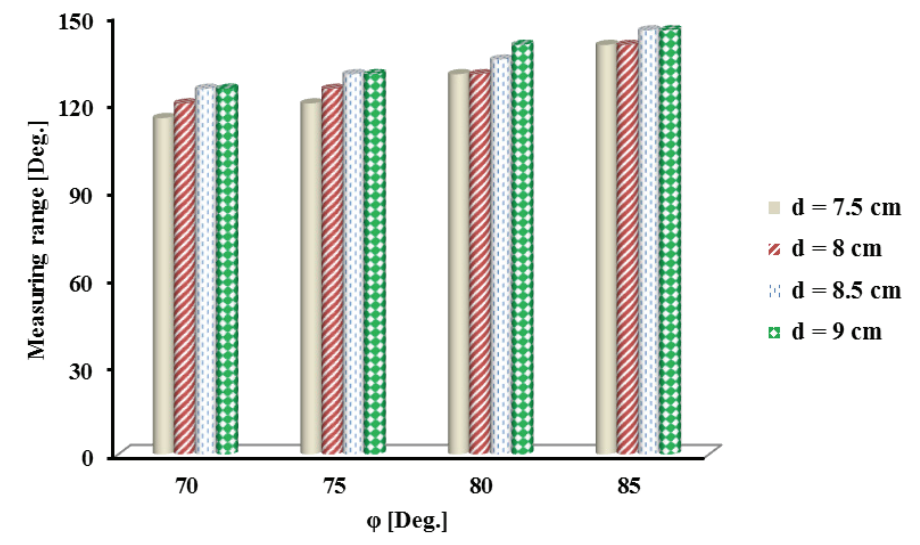

Fig. 5. The change of measuring range caused by the combination of two variables $(d$ and $\varphi)$.

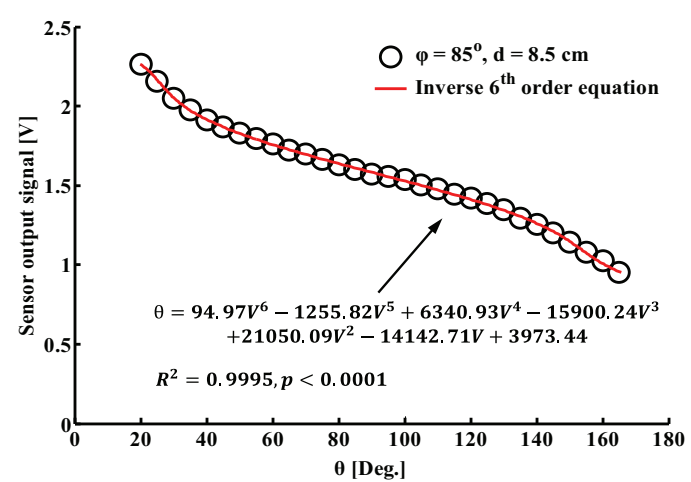

(a) Inverse modeling

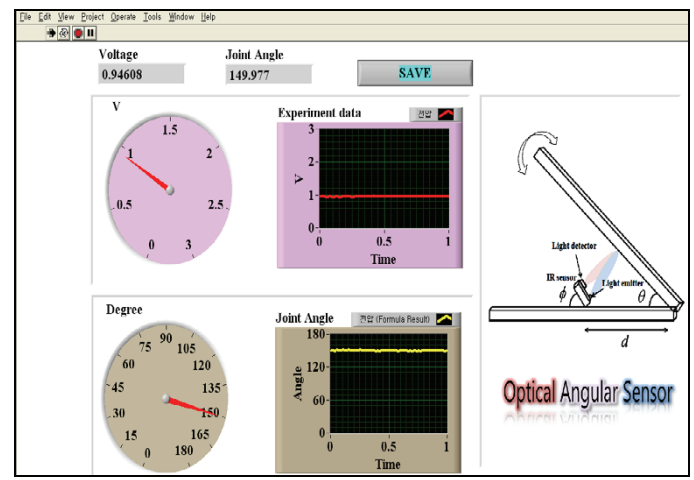

(b) LabVIEW display

Fig. 6. Inverse model with the polynomial equation and the joint angle display platform.

Figure 6(a) shows the inverse modeling over $\theta=20^{\circ} \sim 165^{\circ}$ with $\varphi=85^{\circ}$ and $d=8.5 \mathrm{~cm}$ for joint angle measurements. The inverse $6^{\text {th }}$ order equation showed the best fit in test range. Only monotonically decreasing region was used to get inverse model directly to acquire joint angle information. As the result of regression process, the inverse polynomial equation is statistically significant $\left(R^{2}=0.9995\right.$ with $p<$ 0.0001). Figure 6(b) shows the example of LabVIEW display of calculated joint angle. We can directly monitor the joint angle information with detected light signal in real time.

The suggested optical sensor was evaluated by comparing with commercial goniometer (PS-2137, PASCO, USA) and 3D motion capture system (Eagle, Motion analysis, USA). Figure 7 shows the relationship between the reference joint angle and the measured angle with other commercial sensors. The measured angles are correlated well with reference joint angle. Experiment results show that the proposed sensor has the root-mean-square error (RMSE) of $\pm 0.1275^{\circ}$. The result describes that the developed joint angle sensor is comparable with other angle measurement tools.

Guidelines for normal range of motion have been suggested by American Academy of Orthopaedic Surgeons (AAOS). The AAOS represent the normal range of motion of the human joints. Knee joint extension varies from $0^{\circ} \sim 10^{\circ}$ and flexion from $0^{\circ} \sim 135^{\circ}$. Wrist joint extension varies from $0^{\circ} \sim$ $70^{\circ}$ and flexion from $0^{\circ}$ to $80^{\circ}$. The ankle dorsiflexion is between $0^{\circ} \sim 20^{\circ}$ and plantarflexion from $0^{\circ}$ $\sim 50^{\circ}$. Hip joint extension varies from $0^{\circ} \sim 30^{\circ}$ and flexion from $0^{\circ} \sim 120^{\circ}$. Similarly, for the elbow 


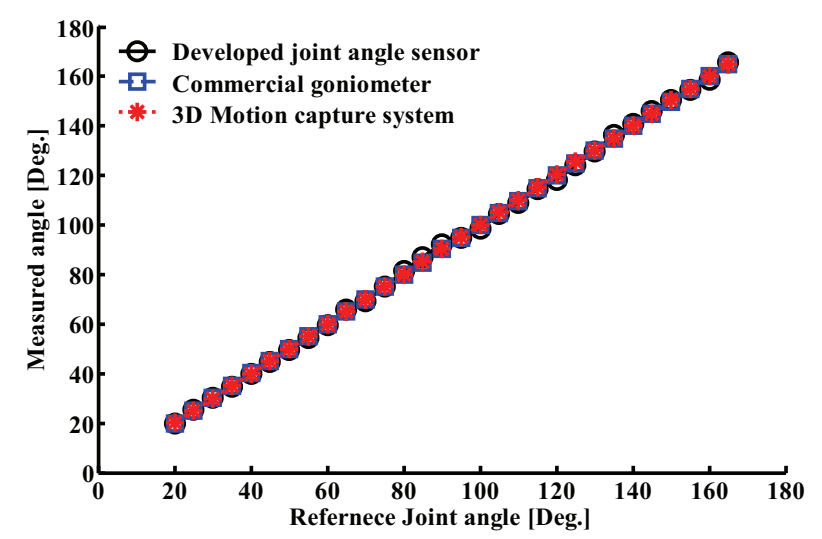

Fig. 7. Measured angle with suggested joint angle sensor, commercial goniometer, and 3D motion capture system versus reference joint angle.

extension is 0 degree and flexion from $0^{\circ} \sim 150^{\circ}$ [11|12].

The developed joint angle sensor is valid in the measuring range of $\theta=20^{\circ} \sim 165^{\circ}$. The measuring range can be modified from $0^{\circ} \sim 145^{\circ}$ by changing the reference point. Further study will be done to increase the measuring range up to $180^{\circ}$. Also, we will study the optimal design of the optical angular sensor to be attached on the human body.

\section{Conclusions}

In this study, we developed an optical system for monitoring of human joint movements. Many experiments with various parameters were performed to find the optimal combination for joint angle measurements. The effect of the attached distance and the attached angle on the joint angle was investigated to get accurate and wide range of joint angle information. In general, as the attached distance and the attached angle are increased, the measuring range of joint angle is increased. However, excessive attached sensor's angle was not suitable for joint angle measurement due to insufficient resolution. The best combination of parameters was applied to find the appropriate inverse mathematical model. The joint angle change can be detected in real time with LabVIEW display. The accuracy of the developed sensor was verified by comparing with commercial goniometer and 3D motion capture system. The measured joint angle was correlated well with the reference joint angle. This study can be applied to rehabilitation and sport science fields.

\section{Acknowledgements}

This research was supported by Basic Science Research Program through the National Research Foundation of Korea (NRF) funded by the Ministry of Education, Science and Technology (No. 20100023158).

\section{References}

[1] Kim YS, Jang SW, Yoo YS. Mobil assessment system for shoulder joint rehabilitation: System development and preliminary study. IJBSBT. 2014; 6(2): 51-60. 
[2] Yamamoto I, Inagawa N, Matsui M, Hachisuka K, Wada F, Hachisuka A. Research and development of compact wrist rehabilitation robot system. Bio-Med. Mater. Eng. 2014; 24(1): 123-128.

[3] Lea RD, Gerhardt JJ. Current concepts review range-of-motion measurements. J. Bone. Joint. Surg. Am. 1995; 77(5): 784-798.

[4] Luinge HJ, Veltink PH. Inclination measurement of human movement using a 3-D accelerometer with autocalibration. IEEE Trans. Neural. Syst. Rehabil. Eng. 2004; 12(1): 112-121.

[5] Jung GI, Kim JS, Lee TH, Choi JH, Oh HB, Kim AH, et al. Development of an optical fiber sensor for angular displacement measurements. Bio-Med. Mater. Eng. 2014; 24(1): 771-780.

[6] Mohamed AA, Beyea J, Landry J, McGibbon CA, Sexton A, Baba J. Comparison of strain-gage and fiber-optic goniometry for measuring knee kinematics during activities of daily living and exercise. J. Biomech. Eng. 2012; 134(8): 084502 .

[7] Noh DK, Lee NG, You JH. A novel spinal kinematic analysis using X-ray imaging and vicon motion analysis: A case study. Bio-Med. Mater. Eng. 2014; 24(1): 593-598.

[8] Steele BG, Belza B, Cain K, Warms C, Coppersmith J, Howard J. Bodies in motion: monitoring daily activity and exercise with motion sensors in people with chronic pulmonary disease. J. Rehabil. Res. Dev. 2003; 40(5): 45-58.

[9] Stupar DZ, Bajic JS, Manojlovic LM, Slankamenac MP, Joza AV, Zivanov MB. Wearable low-cost system for human joint movements monitoring based on fiber-optic curvature sensor. IEEE Sens. J. 2012; 12(12): 3424-3431.

[10] Chao KH, Tsai JC. Surface tension and concentration measurement of sub-L solution using a cantilever-based optical gauging system. IEEE J. Quan. 2010; 46(9): 1268-1274.

[11] Roaas A, Andersson GB. Normal range of motion of the hip, knee and ankle joints in male subjects, 30-40 years of age. Acta. orthop. scand. 1982; 53(2): 205-208.

[12] Norkin CC, White DJ. Measurement of Joint Motion: A Guide to Goniometry. Philadelphia, FA Davis; 2009. 